\title{
High-energy Picosecond Laser Pulse Recirculation for Compton Scattering
}

I. Jovanovic, S. G. Anderson, S. M. Betts, C. Brown, D.

J. Gibson, F. V. Hartemann, J. E. Hernandez, M.

Johnson, D. P. McNabb, M. Messerly, J. Pruet, M. Y. Shverdin, C. W. Siders, A. M. Tremaine, C. P. J. Barty

June 14, 2007

Particle Accelerator Conference ' 07

Albuquerque, NM, United States

June 25, 2007 through June 29, 2007 
This document was prepared as an account of work sponsored by an agency of the United States Government. Neither the United States Government nor the University of California nor any of their employees, makes any warranty, express or implied, or assumes any legal liability or responsibility for the accuracy, completeness, or usefulness of any information, apparatus, product, or process disclosed, or represents that its use would not infringe privately owned rights. Reference herein to any specific commercial product, process, or service by trade name, trademark, manufacturer, or otherwise, does not necessarily constitute or imply its endorsement, recommendation, or favoring by the United States Government or the University of California. The views and opinions of authors expressed herein do not necessarily state or reflect those of the United States Government or the University of California, and shall not be used for advertising or product endorsement purposes. 


\title{
High-energy Picosecond Laser Pulse Recirculation for Compton Scattering*
}

\author{
I. Jovanovic, S. G. Anderson, S. M. Betts, C. Brown, D. J. Gibson, \\ F. V. Hartemann, J. E. Hernandez, M. Johnson, D. P. McNabb, M. Messerly, \\ J. Pruet, M. Y. Shverdin ${ }^{\dagger}$, A. M. Tremaine, C. W. Siders, C. P. J. Barty \\ Lawrence Livermore National Laboratory, Livermore, CA 94550, USA
}

\begin{abstract}
Frequency upconversion of laser-generated photons by inverse Compton scattering for applications such as nuclear spectroscopy and gamma-gamma collider concepts on the future ILC would benefit from an increase of average source brightness. The primary obstacle to higher average brightness is the relatively small Thomson scattering cross section. It has been proposed that this limitation can be partially overcome by use of laser pulse recirculation. The traditional approach to laser recirculation entails resonant coupling of low-energy pulse train to a cavity through a partially reflective mirror [1]. Here we present an alternative, passive approach that is akin to "burst-mode" operation and does not require interferometeric alignment accuracy. Injection of a short and energetic laser pulse is achieved by placing a thin frequency converter, such as a nonlinear optical crystal, into the cavity in the path of the incident laser pulse. This method leads to the increase of x-ray/gamma-ray energy proportional to the increase in photon energy in frequency conversion. Furthermore, frequency tunability can be achieved by utilizing parametric amplifier in place of the frequency converter.
\end{abstract}

\section{RECIRCULATION INJECTION BY NONLINEAR GATING CONCEPT}

Many applications of high intensity lasers such as Compton-scattering based light sources, high-harmonics generation, laser produced plasmas and Thomson scattering are limited by low conversion efficiencies. The efficiency of nonlinear process induced by interaction of a short intense laser pulse with an optically thin medium could be increased by reusing the laser photons after each interaction. Current pulse recirculation schemes are based either on resonant cavity coupling $[2,3]$ or active (electrooptic) pulse switching $[4,5]$ into and out of the resonator.

Here, we describe an alternative efficient pulse trapping scheme based on nonlinear frequency conversion, termed recirculation injection by nonlinear gating (RING). In the simplest implementation of this technique, the incident laser pulse at the fundamental frequency enters the resonator and is efficiently frequency doubled. The resonator

\footnotetext{
* This work was performed under auspices of the U.S. Department of Energy by University of California, Lawrence Livermore National Laboratory under Contract W-7504-Eng-48.

† shverdin2@1lnl.gov
}

mirrors are dichroic, coated to transmit the $(1 \omega)$ light and reflect the 2nd harmonic (see Fig. 1). The upconverted $2 \omega$ pulse becomes trapped inside the cavity. After many roundtrips, the laser pulse decays primarily due to Fresnel losses at the crystal faces and cavity mirrors. The major advantage of the outlined recirculation scheme compared to active (electro-optic or accousto-optic) pulse switching is that the pulse traverses a significantly thinner optical material. Conversion efficiency, $\eta \propto I L^{2}$, where $I$ is the pulse intensity and $L$ is the crystal thickness. A $1 \mathrm{~mm}$ thick BBO crystal efficiently frequency doubles pulses at incident intensities of $\approx 10 \mathrm{GW} / \mathrm{cm}^{2}$. A typical thickness for a Pockels cell and a waveplate is $\approx 1 \mathrm{~cm}$. For short, high peak power pulses, nearly an order of magnitude decrease in the length of the traversed medium reduces pulse dispersion and nonlinear phase accumulation that ultimately leads to beam break-up. Resonant cavity coupling techniques have so far been demonstrated for low peak power pulses.

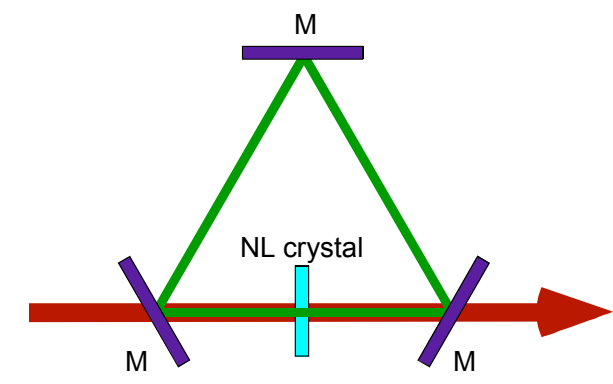

Figure 1: RING cavity principle

\section{EXPERIMENTAL RESULTS}

We have completed a proof of principle experiment which demonstrates the RING scheme [Fig 2(a)]. The experiment was conducted at the Advanced Petawatt Concepts facility at LLNL [6]. The incident pulse was at $1053 \mathrm{~nm}, 10 \mathrm{~Hz}$, chirped from its $250 \mathrm{fs}$ transform limit to $10 \mathrm{ps}$, with a pulse energy of $1 \mathrm{~mJ}$. The spatial profile of the incident beam was nearly gaussian in space, with a spot size of $3 \mathrm{~mm}$ and nearly flat-top in time. A lens telescope collimated the laser beam before the resonator. $80 \mu \mathrm{J}$ of $2 \omega$ light was generated with an anti-reflection (AR) coated $1.5 \mathrm{~mm}$ type I BBO crystal. Flat 1" dichroic cavity mirrors were e-beam coated by Lattice Electro-Optics to achieve 
vendor specified transmission of $>98 \%$ at $1053 \mathrm{~nm}$ and reflection of $>99.7 \%$ at $526.5 \mathrm{~nm}$. A pair of photodiodes monitored the recirculating light by measuring the scatter off the nonlinear crystal and the leakage through one of the cavity mirrors.

The cavity ring-down signal is shown in Fig. 2(b). $2 \omega$ light leaked through one of the cavity mirrors was filtered and measured by a fast photodiode. The signal decays with each roundtrip inside the cavity. In this experiment, we achieved a total signal enhancement of 28.5, calculated as the ratio of the integrated energy of all the roundtrips divided by the energy in the initial $2 \omega$ pulse. Slight modulation of the signal power vs time can be observed in Fig. 2(b). This is due to slight misalignment between the polarization of $2 \omega$ light and the crystal axis. In this experiment, the decay inside the cavity is due to mainly to fresnel and diffraction losses.
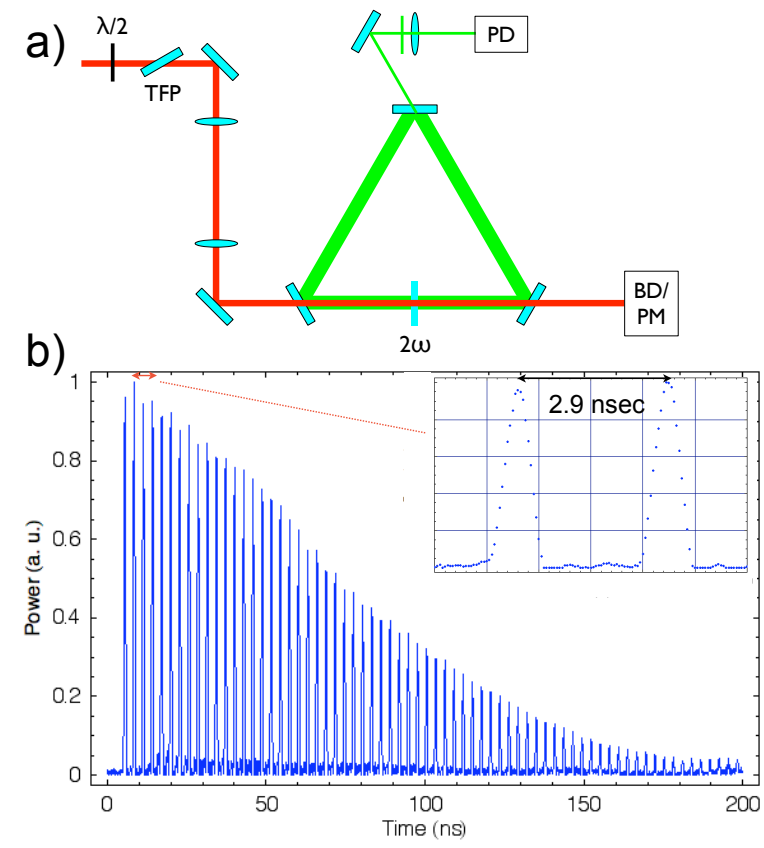

Figure 2: (a) RING cavity experimental set-up and (b) the associated recirculating $2 \omega$ signal in the cavity vs time. The integrated energy is $28.5 \times$ higher than for a single pass. The temporal pulse separation of $2.9 \mathrm{nsec}$ shown in the inset corresponds to the roundtrip time of the cavity.

\section{Simulations}

We simulate the expected performance of the three mirror cavity assuming a reflection of $99.7 \%$ at each mirror, transmission of $99.6 \%$ through the crystal, and crystal thickness of $1 \mathrm{~mm}$. The values for linear crystal absorption and the nonlinear refractive index are estimated as $0.01 \mathrm{~cm}^{-1}$ and $5.5 \times 10^{-16} \mathrm{~cm}^{2} / \mathrm{W}$, respectively. We assume intensity of $10 \mathrm{GW} / \mathrm{cm}^{2}$ in the initial $2 \omega$ pulse and neglect any diffraction losses. We predict cavity enhancement of 71 by integrating the energy versus roundtrips curve of
Fig. 3(a). For these parameters, the nonlinear phase accumulation in the BBO crystal exceeds $3.5 \mathrm{rad}$ after 100 cavity roundtrips. The pulse dispersion is relatively low, as the beam travels through a total of $10 \mathrm{~cm}$ of $\mathrm{BBO}$ crytstal after 100 roundtrips.
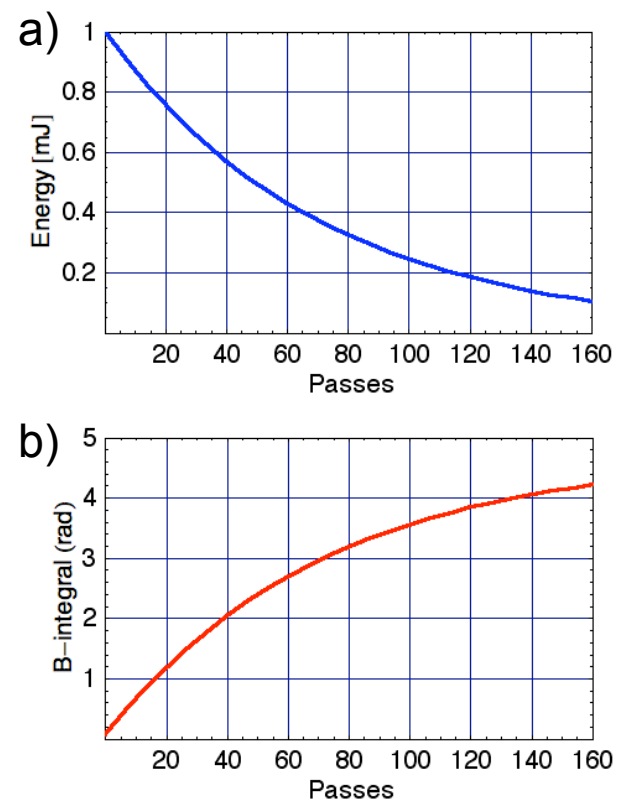

Figure 3: Numerical modeling of 3 mirror RING cavity performance, assuming a total loss of $1.3 \%$ per roundtrip. (a) Pulse energy in the cavity vs number of roundtrips. The total predicted cavity enhancement is 71 . (b) Nonlinear phase accumulation versus the number of roundtrips.

\section{Cavity designs for Compton-scattering}

The primary motivation behind the RING technique is the average brightness enhancement of high peak power $\gamma$-rays generated by Compton-backscattering laser photons off a relativistic electron beam. This Comptonbackscattering based light source ((T-REX) is currently under construction at LLNL [8]. Specifications for the RING cavity include a focal spot of $20 \mu \mathrm{m}$, peak intensity of $10^{14} \mathrm{~W} / \mathrm{cm}^{2}$, laser pulse duration of $10 \mathrm{ps}$, and total pulse energy of $500 \mathrm{~mJ}$ at $532 \mathrm{~nm}$. In addition, the optical cavity must be robust, reliable, and simple to align and operate. Our RING cavity design shown in Fig. 4 meets the outlined specifications.

The linear RING cavity consists of two concave mirrors with identical radii of curvature and a nonlinear crystal placed at the focus. The mirror spacing is equal to the sum of the focal lengths of the two mirrors. The cavity is selfimaging, meaning that the ABCD matrix for one roundtrip is $\left(\begin{array}{cc}-1 & 0 \\ 0 & -1\end{array}\right)$. The designed cavity is equivalent to a confocal resonator. The curvature of the incident $1 \omega$ beam is adjusted to produce a collimated wavefront after the first cavity mirror. This produces a collimated $2 \omega$ pulse after 


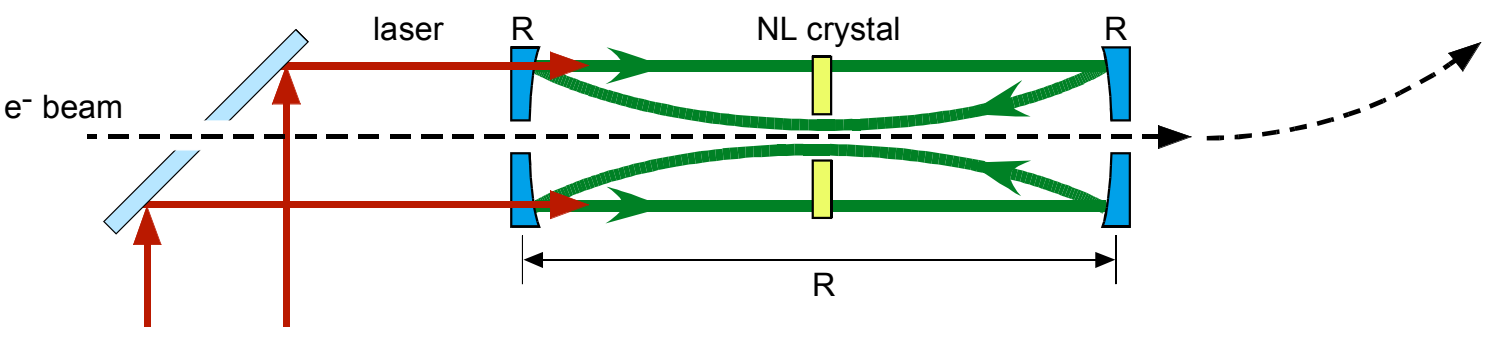

Figure 4: RING cavity design for Compton-backscattering based light source. The entrance and exit mirrors and the nonlinear crystal have a hole for passage of the electron beam.

the nonlinear crystal. The $2 \omega$ beam focuses when traveling from right to left and recollimates when traveling from left to right inside the cavity. The nonlinear crystal has a small hole $(\approx 100 \mu \mathrm{m})$ which is larger than the focal laser spot at its center to reduce the nonlinear phase accumulation, total pulse dispersion, and absorptive and scattering losses. The focal length of the mirrors, and hence the repetition rate of the laser pulses in the cavity will be set to an integer subharmonic of the maximum repetition rate of the arriving electron bunches (currently $2.2 \mathrm{GHz}$ at the LLNL linac). The electron beam passes through a small hole $(\approx 1 \mathrm{~mm})$ in the entrance cavity mirror and leaves through a hole in the exit mirror. The e- beam is steered with external magnets and focuses at the laser focus. $\gamma$-ray generation occurs at the cavity focus in the propagation direction of the electron beam. This RING design can be scaled to higher incident laser powers by increasing the aperture of the cavity mirrors and the nonlinear crystal.

The nonlinear frequency conversion based beam trapping and recirculation concept can be extended to a variety of potential applications. Circularly polarized positron generation proposed at the International Linear Collider (ILC) [9], requires a high flux of circularly polarized gamma-rays. Inverse Compton scattering, which is one of the options considered, requires $\mathrm{kW}$ scale average power lasers with pulse durations of at few picoseconds. RING technology would simplify the requisite laser technology by lowering the average power requirements. The cavity design shown in Fig. 4 can be slightly modified to achieve a circularly polarized laser beam at the cavity focus (Fig. 5). Here, folding the cavity places the nonlinear crystal away from the high energy electron beam; adding two retardation plates produces same handed circularly polarized light at the focus after each roundtrip.

We presented a novel pulse recirculation design suitable for trapping short high peak power pulses. RING recirculation minimizes pulse dispersion and nonlinear phase accumulation which limits the performance of active pulse switching schemes. The RING cavity should provide nearly 2 orders of magnitude improvement in average brightness of Compton-backscattering $\gamma$-ray light source currently being developed at LLNL.

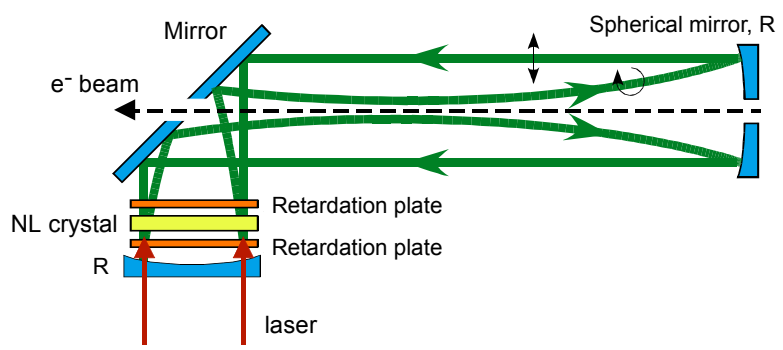

Figure 5: RING cavity design for polarized gamma-ray generation. Here, the cavity is folded to prevent damage to the crystal from the $\mathrm{GeV}$ electron beam. Retardation plates before and after the crystal compensate for the polarization rotation in the crystal and mirror coatings to produce circularly polarized light at the focus.

\section{REFERENCES}

[1] G. Klemz, K. Mönig, I. Will, Nucl. Instrum. Meth. A 564, 212 (2006).

[2] C. Gohle, T. Udem, M. Herrmann, J. Rauschenberger, R. Holzwarth, H. Schuessler, F. Krausz, and T. Hänsch, Nature, 436, 234 (2005).

[3] R. J. Jones, K. D. Moll, M. J. Thorpe, and J. Ye, Phys. Rev. Lett., 94, 193201 (2005).

[4] T. Mohamed, G. Andler, R. Schuch, Opt. Commun. 214, 291 (2002).

[5] D. Yu and B. Stuart, in: Particle Accelerator Conference, PAC'97, Vancouver, DC, Canada, May 12-16, 1997.

[6] I. Jovanovic, C. Brown, B. Wattellier, N. Nielsen, W. Molander, B. Stuart, D. Pennington, and C. P. J. Barty, Rev. Sci. Instrum., 75, 5193 (2004).

[7] P. Chen, D. Bullock, D. Yu, Nucl. Inst. Meth. A 355, 130 (1995).

[8] Lawrence Livermore National Lab, Tech. Rep. UCRL-TR206825, 2004.

[9] F. Bechtel, G. Klämke, G. Klemz, K. Mönig, H. Nieto, H. Nowak, A. Roscam, J. Sekaric, A. Stahl, Nucl. Inst. Meth. A 564, 243 (2006). 